\title{
Source Localization of EM Waves in the Near-Field of Spherical Antenna Array in the Presence of Unknown Mutual Coupling
}

\author{
Oluwole John Famoriji (D) and Thokozani Shongwe \\ Department of Electrical and Electronic Engineering Science, University of Johannesburg, P.O. Box 524, Auckland Park, \\ 2006 Johannesburg, South Africa \\ Correspondence should be addressed to Oluwole John Famoriji; famoriji@mail.ustc.edu.cn
}

Received 12 July 2021; Revised 23 October 2021; Accepted 12 November 2021; Published 14 December 2021

Academic Editor: Fawad Zaman

Copyright (C) 2021 Oluwole John Famoriji and Thokozani Shongwe. This is an open access article distributed under the Creative Commons Attribution License, which permits unrestricted use, distribution, and reproduction in any medium, provided the original work is properly cited.

\begin{abstract}
To obtain an antenna array with isotropic radiation, spherical antenna array (SAA) is the right array configuration. The challenges of locating signals transmitted within the proximity of antenna array have been investigated considerably in the literature. However, near-field (NF) source localization of signals has hitherto not been investigated effectively using SAA in the presence of mutual coupling (MC). MC is another critical problem in antenna arrays. This paper presents an NF range and directionof-arrival (DoA) estimation technique via the direction-independent and signal invariant spherical harmonics (SH) characteristics in the presence of mutual coupling. The energy of electromagnetic (EM) signal on the surface of SAA is captured successfully using a proposed pressure interpolation approach. The DoA estimation within the NF region is then calculated via the distribution of pressure. The direction-independent and signal invariant characteristics, which are $\mathrm{SH}$ features, are obtained using the DoA estimates in the NF region. We equally proposed a learning scheme that uses the source activity detection and convolutional neural network (CNN) to estimate the range of the NF source via the directionindependent and signal invariant features. Considering the $\mathrm{MC}$ problem and using the DoA estimates, an accurate spectrum peak in the multipath situation in conjunction with $\mathrm{MC}$ and a sharper spectrum peak from a unique MC structure and smoothing algorithms are obtained. For ground truth performance evaluation of the SH features within the context of NF localization, a numerical experiment is conducted and measured data were used for analysis to incorporate the MC and consequently computed the root mean square error (RMSE) of the source range and NF DoA estimate. The results obtained from numerical experiments and measured data indicate the validity and effectiveness of the proposed approach. In addition, these results are motivating enough for the deployment of the proposed method in practical applications.
\end{abstract}

\section{Introduction}

Antenna arrays, in which the distribution of radiating elements is over a spherical surface, remain the answer to the isotropic requirements [1]. Spherical antenna arrays (SAA) have the capability of receiving electromagnetic (EM) wave with equal strength regardless of the polarization and the direction-of-arrival (DoA). To take advantage of the highest degree of freedom, the antenna array under consideration must have the ability to determine the DoA and polarization of an incoming EM wave, impinging from all directions on the unit sphere. Although previous theoretical description has been given on SAA [2-6], more in-depth and precise description of the EM characteristics of the SAA remains an open challenge.

Multiple EM signal localization within the proximity of antenna array has been dealt with in the literature. Most works considered the sources that are in far-field (FF) of the array [8-10]. However, this paper focuses on sources that are in the near-field (NF) of SAA; in this case, it will be possible to estimate DoA and range of the source. This has not been considered in the previous works. Apart from EM systems, NF localization also applies to various areas such as sonar systems, seismic exploration, and microphone arrays. In this paper, EM systems utilizing SAA (as shown in Figure 1) [7] are considered. Spherical harmonics (SH) 


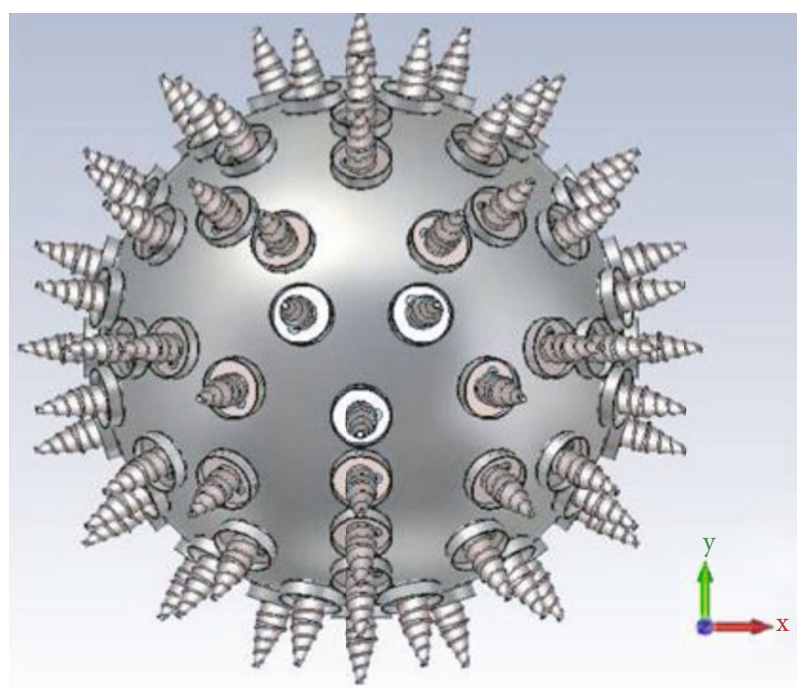

Figure 1: A typical SAA with 64 elements [7].

decomposition can be employed in the representation of reflected signals based on the coefficients, reflector locations, and mode strength matrix [11]. Radial filters have been adopted for the separation of sources, which are situated at various ranges from the system. Some algorithms have also been developed for radial filters $[11,12]$ and NF beamforming $[13,14]$ in the SH domain. However, each of the algorithms requires prior knowledge of the DoA and range of the NF source. The NF source localization of signals has hitherto not been effectively investigated using SAA in a harsh environment. Therefore, presenting an NF range and DoA estimation technique using the direction-independent and signal invariant $\mathrm{SH}$ features towards NF localization of EM signals using SAA becomes crucial.

Recently, Wu and Yan [15] presented a 3-dimensional far-field and near-field source localization via cross array. The method is applicable to different symmetric cross array and disallowed aperture loss. In [16], Shu et al. employed a spatially spread vector sensor to localize 3-D near-field source. This method is applicable to non-free-space propagation models at arbitrarily unknown path-loss exponent and offers an enhanced performance estimation because of the inherent extension of the vector sensor's spatial aperture in spread structure. A mixed near-field and far-field using discrete Fourier transform and orthogonal matching pursuit was used achieve a low complex localization method [10]. This method shows robustness at low SNR and does not demand any matrix decomposition. Furthermore, a onesnapshot mixed far-field and near-field localization algorithm based on discrete fractional Fourier transform is developed [17, 18]. This method simultaneously estimates and classifies mixed sources. Moreover, Kuznetsov et al. [19] performed an autocorrelation analysis and near-field localization of the radiating sources with cyclostationary properties. An autocorrelation analysis procedure for the characterization of radiated emissions from multifunctional digital electric devices in the reactive near-field region is presented and takes advantage of spatial-time localization of transmission line [19].
Furthermore, $\mathrm{Wu}$ and Yan [20] proposed a gridless mixed-source localization method using a low-rank matrix reconstruction. A Vandermonde structure was established for the estimation of DoA and range via the fourth-order cumulants for the development of two spatial matrices that only relate to range and DoA. This approach needs no discretization of range nor the angle regions [20]. Also, an improved near-field multiple signal classification method has been proposed in [21] for DoA estimation accuracy improvement at low SNR. The method improves accuracy by 2 degrees at that low SNR. He et al. [22] proposed a cumulant-based method for multiple near-field source localizations using an exact source-sensor spatial geometry. Here, there is no Fresnel approximation of spatial phase-delay and nonconsideration of propagation attenuation made; i.e., no simplification is made. The algorithm is noniterative, simple, and search-free; it accommodates all arbitrarily unknown propagation loss. In the same vein, Ma et al. [23] investigated the challenge of mixed far-field and near-field source localization by linear electromagnetic-vector-sensor array with phase/gain uncertainties. The estimates are able to generate the unknown gain or phase errors. This technique requires no spectral search and no restriction imposed on the electromagnetic vector sensors placement and realization of more reasonable signal type classification. Linear tripole array [24] and exact spatial propagation geometry [25] concepts have also been used for the realization of mixed nearfield and far-field source localization. Moreover, near-field source localization has been dealt with in different angles using source non-circularity and based on the virtual ESPRIT idea [26], symmetric subarrays [27], two-level nested arrays [28], and subspace [29]. However, no attention has been paid to near-field source localization in spherical domain using antenna array.

There are existing methods reported in the literature for the estimation of DoA such as estimation of subspace rotational in variance technology (ESPRIT) algorithm, multiple signal classification (MUSIC), MUSIC group delay [11, 18, 19], the steered response power with phase transform (SRP-PHAT) [30-35], generalized cross-correlation (GCC) [36], adaptive eigenvalue decomposition [37], onedimensional MUSIC [38], and order aware method [39]. MUSIC has been applied to the $\mathrm{SH}$ structure in NF (MUSIC-SH) [33, 40-42]. Because MUSIC-SH is sensitive to distortion in multipath, Nakamura [43] developed another technique named direct-path dominance (DPD). In NF, mode strengths depend on source range that is not a priori known. As such, DPD is only conducted in the dimension of time, and because of this, MUSIC-SH-DPD requires higher frames [43]. In the application of NF frequency smoothing, normalizing a source that is range dependent is needed. The other DoA estimation method formulated in the SH domain is the minimum variance distortionless response [44].

Some works on NF localization have been presented in the literature. He et al. [45] addressed the problem of partial NF EM source localization using an array of cross-dipole. An algorithm that is based on maximum likelihood was developed for the estimation of range and angle parameters. 
The search over the polarization parameters is not required in the algorithm [45]. Guzey et al. [46] presented the localization of NF radio-controlled devices under a multipath environment using a uniform linear array (ULA). The authors developed a smooth 2-D MUSIC NF localization framework for radio control devices in a multipath fading event. The performance evaluation of symmetric subarraybased NF localization and 2-D MUSIC was compared with the developed smooth 2-D MUSIC framework [46]. Recently, Zheng et al. [47] proposed a source localization framework for mixed NF and FF sources using symmetric double nested antenna arrays. In FF sources, the DoAs are estimated using 1-D MUSIC, and the NF part was extracted from the FF using the oblique projection method. In the end, the range estimates of NF sources were obtained from the NF DoA estimates using 1-D peak searching method. The developed algorithm used the large coarray aperture to enhance the localization performance and capable of accurately differentiating the kind of sources under consideration [47].

Another NF localization has been more recently reported in [48]. The authors presented a comprehensive and generalized method for the evaluation of spatiotemporal energy that is localized in the proximity of antenna arrays of arbitrary structures and excitation of signals. Finitedifference time-domain approach was employed to compute the pointing localized energy for all structures by special consideration on the calculated fields as against the currents of the source. It is shown and demonstrated that the localized energy is fundamentally different from the classical antenna reactive energy, associated with frequency-domain Q-factors of electrically small structures. The developed concept of localized energy and the techniques developed for the investigation of its relevant new data help in the implementation of the ultrahigh gain closely packed antennas, which are useful for the emerging $5 \mathrm{G}$ communications. Ma et al. [49] also presented an algorithm for mixed FF and NF source localization using a linear tripole antenna array. The authors formulated special fourth-order cumulants and utilized the shift-invariant properties in cumulant domain to estimate the source parameters. The resulted estimate is used to simplify the cyclically ambiguous estimate and consequently generate parameter estimates with high accuracy.

Furthermore, with the current growth in technology and system applications, systems are becoming smaller, leading to smaller interelement distance in an array. This causes strong mutual coupling, impedance mismatch, and poor radiation characteristics. This problem will often affect signal antenna array processing and more severe in the near field.

Lately, Zheng et al. [50] proposed a new symmetric nonuniform linear array, named symmetric displaced coprime array (SDCA), for simultaneous localization of NF and FF sources. The SDCA is only decided via closed-form expression, and the corresponding coarray ranges are calculated by analysis. Besides, two optimum SDCA structures were formulated by maximizing the numbers of the unique and consecutive lags in difference coarray. The proposed SDCA configuration shows some levels of superiorities over the existing symmetric nonuniform linear arrays. Therefore, so far, the challenges of locating signals transmitted within the proximity of antenna array have been investigated considerably in the literature. However, NF source localization of signals has hitherto not been investigated effectively using SAA in a harsh environment.

From the localization methods discussed above, it is evident that most reported works only focused on FF sources. However, in NF $[1,44-50]$, the assumption that the waveform is planar, is not always accurate; this consequently causes a high error margin in practice. For accurate antenna array processing, the spherical wavefront from NF sources requires adequate consideration [51]. Although there are some works on NF localization, only a few reported experimental data, which is the true test of any procedure and concept.

In addition, for NF sources, some techniques have been reported in the literature for the estimation of range [40, 52-54] and DoA [7, 9-31, 45, 55]. There is a noticeable variation in the received energy at various observation points in NF EM wave propagation, because of the change in distance that exists between the source and point of observation. However, in the case of FF EM wave propagation, the distance of the source is negligible as the EM energy of the received signal at various observation points has infinitesimal or no variation. The traditional techniques for estimation of NF DoA did not take advantage of this feature of the NF EM signal propagation. For the case of range estimation, most approaches considered the environment ideal. There is performance degradation of the approaches in noisy and harsh environments because the mode strengths are sensitive to the surrounding. Furthermore, the required frequency range for consideration in the estimation of source range is a function of the same source range. Hence, a learning-based technique is more appropriate for the accurate estimation of the source range.

In contrast to the reports in the literature, the key innovation and major contributions in this article are summarized as follows. This paper presents an NF range and DoA estimation technique via the direction-independent and signal invariant SH characteristics. The energy of EM signal on the surface of SAA is successfully captured using a proposed pressure interpolation approach. The DoA estimation within the NF region is then calculated via the distribution of pressure. The direction-independent and signal invariant characteristics, which are $\mathrm{SH}$ features, are obtained using the DoA estimates in the NF region. We obtained the signal invariant features using $\mathrm{SH}$ coefficient normalization with a particular component, which corresponds to the signal strength of the source. Furthermore, we obtained the rotation-independent features by two approaches: (a) rotation of SH functions on a sphere is conducted by Wigner$\mathrm{D}$ function, whereas on the other hand (b) the impact of DoA is calibrated by the normalization of $\mathrm{SH}$. We equally proposed a learning scheme that uses the source activity detection and CNN to estimate the range of the source of the NF via the direction-independent and signal invariant features. Finally, to the best knowledge of the authors, this 
is the first study on NF source localization of EM waves that considers SAA.

The rest of this article is sectioned as follows. The SHbased signal model and the NF criterion are presented in Section 2. Section 3 presents the proposed NF DoA estimation, while the proposed $\mathrm{CNN}$-based range estimation procedure is given in Section 4. Section 5 presents the algorithm with a systematic implementation procedure. Performance evaluation and comparison between the proposed method and previous methods using experimental measured data are given in Section 6. Finally, Section 7 draws the conclusion.

\section{Signal Model}

Let us consider an EM signal scenario with an SAA of radius $r_{e}$ and $M$ number of antenna elements with associated source positioned at $\left(r_{s}, \theta_{s}, \phi_{s}\right)$ radiating and EM wave $z(k)$ , where $r_{s}$ denotes the radial distance and $\left(\theta_{s}, \phi_{s}\right)$ represents the spherical coordinates [56]. The EM signal pressure observed at $\left(r_{e}, \theta, \phi\right)$ is defined as [57]

$p\left(k, r_{e}, \theta, \phi\right)=\sum_{n=0}^{N} \sum_{m=-n}^{n} b_{n}^{s}\left(k, r_{e}, r_{s}\right)\left[Y_{n}^{m}\left(\theta_{s}, \phi_{s}\right)\right]^{*} Y_{n}^{m}(\theta, \phi) \mathrm{z}(k)$

where $k$ denotes the wave number directly proportional to frequency $f$, i.e. $k=2 \pi f / c, c$ represents speed of the propagating wave, a constant usually taken as the speed of light, and $n$ and $m$ are the order and degree, respectively; while $Y$ denotes harmonics. The symbol $[\cdot]^{*}$ denotes complex conjugate representation. Sound and electromagnetic waves carry energy from one place to another and have a wavelength, frequency, and velocity. However, the velocity of sound waves is much lower than that of electromagnetic waves. Hence, $v=f \lambda$ relationship holds for both. Component of noise can be incorporated into Equation (1) to model the signal and element noise. For ease of derivation, the noise component is not included and not needed while deriving the algorithm. Also, a truncation order is strictly ensured to prevent the problem of aliasing because few $\mathrm{SH}$ order is needed in spatial sampling, which is related to the limited bandwidth in time domain sampling [58]. The boundary of the sphere is a function of $b_{n}^{s}\left(k, r_{e}, r_{s}\right)$ and has a relation $b_{n}\left(k r_{e}\right)$ as

$$
b_{n}^{s}\left(k, r_{e}, r_{s}\right)=i^{-(n-1)} k b_{n}\left(k r_{e}\right) h_{n}\left(k r_{s}\right)
$$

where $n$ represents the order of SAA and $i=\sqrt{-1}$. It shows the radial characteristic of point sources can be estimated using spherical Hankel function $h_{n}\left(k r_{s}\right) \cdot b_{n}\left(k r_{e}\right)$ for a rigid sphere expressed as

$$
b_{n}\left(k r_{e}\right)=4 \pi i^{n}\left[j_{n}\left(k r_{e}\right)-\frac{j_{n}^{\prime}\left(k r_{e}\right)}{h_{n}^{\prime}\left(k r_{e}\right)} h_{n}\left(k r_{e}\right)\right],
$$

where $j_{n}$ is the spherical Bessel function of the first kind and $h_{n}$ is the Hankel function of the second kind, while $j_{n}^{\prime}$ and $h_{n}^{\prime}$ denote their corresponding derivatives. $Y_{n}^{m}(\theta, \phi)$ represents the $\mathrm{SH}$ of $n$ order and $m$ degree expressed as

$$
Y_{n}^{m}(\theta, \phi)=\sqrt{\frac{2 n+1}{4 \pi}} \frac{(n-m) !}{(n+m) !} P_{n}^{m}(\cos \theta) e^{i m \phi},
$$

where $P_{n}^{m}(\cdot)$ denotes the associated Legendre equations. The spatial domain, $p\left(k, r_{e}, \theta, \phi\right)$, transformation to $\mathrm{SH}$ domain $p_{n m}\left(k r_{e}\right)$ is [57]

$$
p\left(k, r_{e}, \theta, \phi\right)=\sum_{n=0}^{N} \sum_{m=-n}^{n} p_{n m}\left(k r_{e}\right) Y_{n}^{m}(\theta, \phi)
$$

By juxtaposing Equations (1) and (5), the coefficient of the $\mathrm{SH}$ can be expressed as

$$
p_{n m}\left(k r_{e}\right)=b_{n}^{s}\left(k, r_{e}, r_{s}\right)\left[Y_{n}^{m}\left(\theta_{s}, \phi_{s}\right)\right]^{*} z(k) .
$$

This holds for an ideal environment where a component of the direct path exists between the antenna and the source. However, in a practical situation, a harsh environment where multipath components exist requires special consideration because of reflections. The multipath feature can be added to Equation (6), by incorporating a reflection parameters $\mathfrak{R}$, which is defined as

$$
\Re=\left\{\left(a_{1}, r_{1}, \theta_{1}, \phi_{1}\right),\left(a_{2}, r_{2}, \theta_{2}, \phi_{2}\right),\left(a_{\varpi}, r_{\varpi}, \theta_{\varpi}, \phi_{\varpi}\right)\right\},
$$

where $\omega$ represents the number of reflections. $a_{l}$ is the reflection coefficient and $\left(r_{l}, \theta_{l}, \phi_{l}\right)$ is the reflection point that corresponds to $g$ th reflection. The relationship between $k$ and $\omega$ is not considered in this paper. Equation (6) can then be written as [58]

$$
\begin{aligned}
p_{n m}\left(k r_{e}\right)= & b_{n}^{s}\left(k, r_{e}, r_{s}\right)\left[Y_{n}^{m}\left(\theta_{s}, \phi_{s}\right)\right]^{*} z(k) \\
& +\sum_{l=1}^{\varrho} b_{n}^{s}\left(k, r_{e}, r_{g}\right)\left[Y_{n}^{m}\left(\theta_{g}, \phi_{g}\right)\right]^{*} e_{g} z(k) .
\end{aligned}
$$

Evidently, based on Equation (8), $p_{n m}\left(k r_{e}\right)$ depends on $r_{s}$ , $\left(\theta_{s}, \phi_{s}\right), z(k)$, and $\mathfrak{R}$. The relationship is given as

$$
p_{n m}\left(k r_{e}\right)=v\left(r_{s}, \Re,\left(\theta_{s}, \phi_{s}\right) z(k)\right),
$$

where $v(\cdot)$ is a variable representing the existing relationship between $p_{n m}\left(k r_{e}\right)$ and parameters. The dependency of $r_{e}$ and $k$ is not discussed exclusively, while the dependency of $p_{n m}$ $\left(k r_{e}\right)$ and the importance is later discussed in Section 4.2.

The FF and NF for SAA can be defined according to their capability to differentiate various source distances from the antenna array. The criterion of the NF defines the distance from which the radial magnitude of signal pressure of a point source is identical to the plane wave [12]. An approach used to determine the criterion of NF is already reported in the literature [37]. Considering a source range $r_{s}$ and an SAA with $N$ order and $r_{e}$ radius, when $k r_{s}>>n$, 
the mode strength of the NF, $b_{n}^{s}\left(k, r_{e}, r_{s}\right)$ is

$$
b_{n}^{s}\left(k, r_{e}, r_{s}\right) \approx(-i)^{(n+1)} b_{n}\left(k r_{e}\right) \frac{e^{i k r_{s}}}{r_{s}} .
$$

Therefore, when $k r_{s}>>n, b_{n}^{s}\left(k, r_{e}, r_{s}\right)$ and $b_{n}\left(k r_{e}\right)$ share similar characteristics [37]. Because the discrimination of source distance is conducted only on source distance itself, the NF criterion is

$$
r_{\mathrm{NF}} \approx \frac{N}{k}
$$

Because the source is outside of SAA, $r_{s}$ is required to satisfy the $r_{s}>r_{e}$ condition. Therefore, for FF and NF, the range of $r_{s}$ is expressed as

$$
\begin{gathered}
\text { Near-field : } r_{e}<r_{s}<\frac{N}{k}, \\
\text { Far-field }: r_{s}>\frac{N}{k} .
\end{gathered}
$$

Because the aim of this work centrals on NF localization, subsequently, only NF source scenario is considered.

\section{DoA Estimation from NF Source}

Estimating the DoA in the FF region, the phase of the received signal at multiple elements contributes to the estimation of DoA when the elements are in close proximity (NF) [59]. The magnitude of the received signal is very similar to various elements for the FF source. In NF scenario, the variation of the received energy at different observation points on the SAA is significant. Using inverse spherical Fourier transform (SFT) on the energy distribution variation on SAA via interpolation, (a) there is variation in the distribution of energy over SAA from its peak at the corresponding point to the DoA and a minimum opposite the maxima, and (b) the profile of the location of maximum energy corresponds to DoA can be estimated via various experimental cases, such as SNR, DoA, and $r_{s}$ [47].

The above points gave rise to the method that NF DoA can accurately and simply be estimated at the point where the highest energy is received on the SAA. It is practically impossible to have elements situated over the whole sphere to understand the distribution of energy for DoA estimation. This problem is solvable via observation interpolation over the whole sphere using spatial sampling and inverse SFT.

The SH coefficients $p_{n m}\left(k r_{e}\right)$ are obtained from the measurements at the position of the elements over the sphere. Using SFT, the signal pressure at an observation point on the sphere can be computed using inverse SFT as

$$
p\left(k, r_{e}, \theta_{e}, \phi_{e}\right)=\sum_{n=0}^{N} \sum_{m=-n}^{n} p_{n m}\left(k r_{e}\right) Y_{n}^{m}\left(\theta_{e}, \phi_{e}\right) \forall\left(\theta_{e}, \phi_{e}\right) \in Z^{2} .
$$

The antenna element closest to the source gets larger EM intensity than the farther elements in wave propagation scenario. Therefore, the DoA estimation can be performed at the point where the highest energy is received on the sphere. This is expressed as

$$
\left(\widehat{\theta}_{s}, \widehat{\phi}_{s}\right)=\arg \max _{\left(\theta_{e}, \phi_{e}\right)}\left|p\left(k, r_{e}, \theta_{e}, \phi_{e}\right)\right|^{2}
$$

For time-dependent signals, the method can be applied by finding the total energy on the accessible frames and applicable frequency bins. DoA estimate is the point where maximum energy is received and can be expressed as

$$
\left(\widehat{\theta}_{s}, \widehat{\phi}_{s}\right)=\arg \max _{\left(\theta_{e}, \phi_{e}\right)} \sum_{\forall \tau} \sum_{\forall k_{f}}\left|p\left(\tau, k_{f}, r_{e}, \theta_{e}, \phi_{e}\right)\right|^{2},
$$

where $k_{f}$ and $\tau$ represent the frequency bin and frame index, respectively.

\section{Range Estimation of NF Source}

This section presents the process involved in extracting signal invariant (SI) and direction-independent (DI) (SIDI) features in spherical domain towards the estimation of range via the DoA estimates obtained from the proposed inverse SFT technique.

4.1. SIDI Feature Extraction. The parameters on which $\mathrm{SH}$ coefficients $p_{n m}\left(k r_{e}\right)$ are dependent have been given in the signal model in Equation (9) to be $p_{n m}\left(k r_{e}\right)=v\left(r_{s}, \mathfrak{R}\right.$, $\left.\left(\theta_{s}, \phi_{s}\right), z(k)\right)$. The work here is to reduce the dependency of $p_{n m}\left(k r_{e}\right)$ on parameters that are not wanted through transformation, which are $\mathfrak{R},\left(\theta_{s}, \phi_{s}\right)$, and $z(k)$. It is frequently challenging to calculate $\mathfrak{R}$ within the context of an enclosure. Note that in a practical situation, there is the existence of scattering, noise, etc. Since, $p_{n m}\left(k r_{e}\right)$ dependency on $\mathfrak{R}$ cannot be established effectively; it is achievable through learning obtained from the training. The SH coefficient $p_{n m}$ $\left(k r_{e}\right)$ transformation problem can be mathematically formulated as

find $T$,

$$
\begin{aligned}
\text { s.t. } \quad q_{n m}\left(k r_{e}\right) & =T\left(p_{n m}\left(k r_{e}\right)\right) \\
q_{n m}\left(k r_{e}\right) & =\tilde{v}\left(r_{e}, \mathfrak{R}\right),
\end{aligned}
$$

where $q_{n m}$ denotes the wanted SIDI features, $T$ represents the transformation on $p_{n m}$, which is unknown to compute $q_{n m}$, and the $\tilde{v}(\cdot)$ denotes the function (unknown) showing the nature of dependency on $\left(r_{e}, \mathfrak{R}\right)$ parameters alone. The approach needed to get the appropriate transformation is discussed as follows.

4.2. SI Features. The SI characteristics must be invariant to the variation of the signal strength $z(k)$ of the source. This is achievable using the $\mathrm{SH}$ coefficient that corresponds to zero-order and zero degrees. Since $Y_{0}^{0}(\theta, \phi)=\sqrt{1 / 4 \pi}$ from 
Equation (4); the $p_{00}\left(k r_{e}\right)$ in Equation (8) is expressed as

$$
p_{n m}\left(k r_{e}\right)=\sqrt{\frac{1}{4 \pi}}\left[b_{0}^{s}\left(k, r_{e}, r_{s}\right)+\sum_{l=1}^{\omega} b_{0}^{s}\left(k, r_{e}, r_{g}\right) e_{g}\right] z(k) .
$$

As shown, $p_{00}\left(k r_{e}\right)$ shares a relationship with $z(k)$. Therefore, $p_{00}\left(k r_{e}\right)$ is employed to normalize $p_{n m}\left(k r_{e}\right)$ as

$\tilde{p}_{n m}\left(k r_{e}\right)=\frac{p_{n m}\left(k r_{e}\right)}{p_{00}\left(k r_{e}\right)}=\frac{b_{n}^{s}\left(k, r_{e}, r_{s}\right)\left[Y_{n}^{m}\left(\theta_{s}, \phi_{s}\right)\right]^{*}+\sum_{g=1}^{\infty} b_{n}^{s}\left(k, r_{e}, r_{g}\right)\left[Y_{n}^{m}\left(\theta_{g}, \phi_{g}\right)\right]^{*} e_{g}}{\sqrt{1 / 4 \pi}\left[b_{0}^{s}\left(k, r_{e}, r_{s}\right)+\sum_{g=1}^{\infty} b_{0}^{s}\left(k, r_{e}, r_{g}\right) e_{g}\right]}$.

So, the $\tilde{p}_{n m}\left(k r_{e}\right)$ dependency on different parameters is now formulated to be

$$
\tilde{p}_{n m}\left(k r_{e}\right)=\widehat{v}\left(r_{s}, \Re,\left(\theta_{s}, \phi_{s}\right)\right),
$$

where $\widehat{v}$ is the parameter showing the $\tilde{p}_{n m}\left(k r_{e}\right)$ dependency on the parameters. Therefore, signal invariant features are observed via normalization of SH coefficients with $p_{00}\left(k r_{e}\right)$. The directional independence (DI) is given below.

4.3. DI via Rotation. From Equation (19), it is observed that $\tilde{p}_{n m}\left(k r_{e}\right)$ depends on the DoA of NF source. For learning the mapping function that maps the $\mathrm{SH}$ features to the source range, it is preferable for these features to depend on $r_{s}$ to the largest magnitude. So, DI becomes crucial. Assume $\left(\widehat{\theta}_{s}, \widehat{\phi}_{s}\right)$ to be the DoA estimate of NF computed through inverse SFT technique. If there is a rotation of the coordinate in such a way that the active $\operatorname{DoA}(\theta, \phi)=(0,0)$, then the features are DI. The rotation of $\mathrm{SH}$ is conducted anticlockwisely on Euler angles $\alpha, \beta$, and $\gamma$ around the corresponding $x_{-}, y_{-}$, and $z$-axes. In practice, $\mathrm{SH}$ rotation can be done via multiplication of it $\mathrm{SH}$ coefficients with matrix $\mathbf{D}(\alpha, \beta \gamma)$ modelled with Wigner-D functions using $(\alpha, \beta, \gamma)$ parameters [57]. Furthermore, consider the condition in which $(\alpha, \beta \gamma)=\left(0,-\widehat{\theta}_{s},-\widehat{\phi}_{s}\right)$. Under such condition, the north pole of the sphere aligns to the DoA. The variation of spherical reflection component depends on the DoA, still, and can be established by learning from training samples. Therefore, the kind of transformation to extract the $\mathrm{SH}$ features that are both SI and DI (SH-SIDIR) is expressed as

$$
q_{n m}\left(k r_{e}\right)=\mathbf{D}\left(0,-\widehat{\theta}_{s},-\widehat{\phi}_{s}\right) \tilde{\mathbf{p}}_{n m}\left(k r_{e}\right)
$$

Putting Equation (18) into Equation (20) gives

$q_{n m}\left(k r_{e}\right)=\frac{b_{n}^{s}\left(k, r_{e}, r_{s}\right)\left[Y_{n}^{m}\left(\theta_{s}, \phi_{s}\right)\right]^{*}+\sum_{g=1}^{\infty} b_{n}^{s}\left(k, r_{e}, r_{g}\right)\left[Y_{n}^{m}\left(\theta_{g}, \phi_{g}\right)\right]^{*} e_{g}}{\sqrt{1 / 4 \pi}\left[b_{0}^{s}\left(k, r_{e}, r_{s}\right)+\sum_{g=1}^{\infty} b_{0}^{s}\left(k, r_{e}, r_{g}\right) e_{g}\right]}$,

where $\theta_{g}^{\prime}=\theta_{g}-\theta_{s}, \phi_{g}^{\prime}=\phi_{g}-\phi_{s}$, and $Y_{n}^{m}(0,0)$ from Equation
(4) is

$$
Y_{n}^{m}(0,0)=\sqrt{\frac{2 n+1}{4 \pi} \frac{(n-m) !}{(n+m) !}} P_{n}^{m}(1)
$$

It is seen that the component of the direct path does not depend on the source DoA. It is also possible to obtain DI by $\mathrm{SH}$ division as follows.

4.4. Directional Independence via Division. DI can be established by using the $\mathrm{SH}$ part of DoA estimated to divide the $\tilde{p}_{n m}\left(k r_{e}\right)$ that corresponds to it. The SI and DI SH features (SH-SIDID) are obtained from

$$
q_{n m}\left(k r_{e}\right)=\tilde{\mathbf{p}}_{n m}\left(k r_{e}\right) \Theta\left[\mathbf{Y}_{n}^{m}\left(\theta \wedge_{s}, \phi \wedge_{s}\right)\right]^{*}
$$

where $\Theta$ is the element-by-element division. Putting $\tilde{p}_{n m}(k$ $r_{e}$ ) of Equation (18) into Equation (23), then

$$
q_{n m}\left(k r_{e}\right)=\frac{b_{n}^{s}\left(k, r_{e}, r_{s}\right)+\sum_{g=1}^{\infty} b_{n}^{s}\left(k, r_{e}, r_{g}\right)\left(\left[Y_{n}^{m}\left(\theta_{g}, \phi_{g}\right)\right]^{*} /\left[Y_{n}^{m}\left(\theta \wedge_{s}, \phi \wedge_{s}\right)\right]^{*}\right) e_{l}}{\sqrt{1 / 4 \pi}\left[b_{0}^{s}\left(k, r_{e}, r_{s}\right)+\sum_{g=1}^{\infty} b_{0}^{s}\left(k, r_{e}, r_{g}\right) e_{g}\right]} .
$$

It is seen now that the component of the direct path does not depend on the source DoA. Note that divisor $Y_{n}^{m}\left(\widehat{\theta}_{s}, \widehat{\phi}_{s}\right)$ could be ill-conditioned depending on the value of $Y_{n}^{m}\left(\widehat{\theta}_{s}\right.$, $\left.\widehat{\phi}_{s}\right)$. Under such a scenario, the impact of the component of the direct path to observation, $b_{n}^{s}\left(k, r_{e}, r_{s}\right)\left[Y_{n}^{m}\left(\theta_{s}, \phi_{s}\right)\right]^{*}$, is minimal, and the level of $q_{n m}\left(k r_{e}\right)$ can be put in a threshold to mitigate the impact. Hence, $q_{n m}\left(k r_{e}\right)$ dependency can be given as

$$
q_{n m}\left(k r_{e}\right)=\tilde{v}\left(r_{s}, \Re\right)
$$

4.5. SH-SIDID versus SH-SIDIR. Here, we state the difference in summary between SH-SIDID and SH-SIDIR features. In the extraction process of the features, we obtain the DoA $\left(\widehat{\theta}_{s}, \widehat{\phi}_{s}\right)$ estimate and signal invariant SH features $\tilde{\mathbf{p}}_{n m}\left(k r_{e}\right)$ first. It is seen from Equation (21) that the effective DoA of SH-SIDIR is $(0,0)$. We can also be ascertained from Equation (24) for SH-SIDID that the impact of $Y_{n}^{m}\left(\theta_{s}, \phi_{s}\right)$ is calibrated using $Y_{n}^{m}\left(\widehat{\theta}_{s}, \widehat{\phi}_{s}\right)$. Therefore, SH-SIDID defines mode strengths, which makes it a better choice for range information representation.

4.6. CNN-Based NF Estimation of Range. For range estimation, both SH-SIDID and SH-SIDIR features have been shown and proven above to possess the required information. The next step is the representation of the features for $\mathrm{CNN}$ [47] in such a way that the learning of the mapping function from the features to their respective range can be achieved. Due to the fact that the information of the source range is associated with mode strengths, $q_{n m}\left(k r_{e}\right)$ magnitude is given to be the input of the CNN. If $N$ defines the SAA order under consideration and $N_{k}$ represents the frequency bins, then, the feature set is in a rectangular form whose 


$$
\begin{aligned}
& \text { dimension is }\left((N+1)^{2}-1\right) \times N_{k} \text { as } \\
& \mathbf{Q}=\left[\begin{array}{cccc}
\left|q_{-11}\left(k_{1} r_{e}\right)\right| & \left|q_{-11}\left(k_{2} r_{e}\right)\right| & . & \left|q_{-11}\left(k_{N_{k}} r_{e}\right)\right| \\
\left|q_{01}\left(k_{1} r_{e}\right)\right| & \left|q_{01}\left(k_{2} r_{e}\right)\right| & . & \left|q_{01}\left(k_{N_{k}} r_{e}\right)\right| \\
. . & . & . \cdot & . . \\
\left|q_{N N}\left(k_{1} r_{e}\right)\right| & \left|q_{N N}\left(k_{2} r_{e}\right)\right| & . & \left|q_{N N}\left(k_{N_{k}} r_{e}\right)\right|
\end{array}\right] .
\end{aligned}
$$

We apply $\log$ transformation to the features before feeding it into the CNN for estimation of range. The realizable source range is divided into a set of particular classes, and the function of the CNN is feature classification to a particular class via computation of the posterior probability $P_{r}\left(r_{s}\right.$ IQ) of feature $\mathbf{Q}$ that belongs to a particular class of $r_{s}$.

4.7. The Architecture of the CNN. We adapted CNN to estimate range via SH-SIDID or SIDIR feature mapping to the range. For the purpose of classification, the source range is divided into many classes. There are seven classes with 10 , $15, \cdots, 40 \mathrm{~cm}$ labels. The network is composed of nine convolutional layers, followed by two fully connected layers. The size and depth of strides present in the convolutional layers are $3 \times 50$ and 4 , respectively. The activation function used for the whole layer is rectified linear unit (ReLU) [55, 60] except for the last layer where the softmax function [61] is employed to obtain the posterior probabilities. We ensured flattened convolutional layers' output prior feeding them into the fully connected layer (the size is 128). The number of the output layer is the same as that of classes, 7 in this scenario. Overfitting is prevented by using $20 \%$ dropout, early stopping with 20 epochs patience level, and validation.

4.8. Range Estimation. Employing the rule of maximum posteriori probability, the range can be computed using

$$
\widehat{r}_{s}=\arg \max _{r_{s}} P_{r}\left(r_{s} \mid \mathbf{Q}\right) .
$$

The range estimates from one frame are not very adequate because the frame may be noisy or has no signal. Therefore, signal activity detection (SAD) is adapted. In [62], Kim and Hahn developed a signal detection scheme via an adaptive context attention model. The model employs context information with the time of waves and deep neural networks. The multiresolution cochleagram-dependent features are provided and used to train the model, and the outputs of the trained model are the signal probability, where we obtain the hard decision label using a threshold. Therefore, SAD is employed to locate the silence frames as well as total range estimates from the signal frames.

\section{Proposed Framework}

This section presents the outline or summary of the whole process involved in the localization of NF source, particularly DoA and range estimation. The block diagram that encapsulates the entire process is as depicted in Figure 2. We used the inverse SFT technique to estimate DoA. SI $\mathrm{SH}$ features are obtained from $\mathrm{SH}$ coefficients. From this DoA estimate, the extraction of the SH-SIDID or SHSIDIR features is performed. Then, the training of CNN is conducted using these features and the labels that correspond to it during training. During testing, the features are fed into the CNN as input to estimate the range successfully. Employing the appropriate frames, we performed posterior probability aggregation, which consequently gives a better range estimation. The step-by-step procedure required to conduct these task is as systematically depicted in Algorithm 1 . The requirements of the algorithm are the observations $p\left(k, r_{e}, \theta, \phi\right)$, a trained SAD model, and trained models with SH-SIDID or SH-SIDIR.

\section{Experiment, Performance Evaluation, Results, and Discussion}

Some works deal with NF source localization of signals using antenna arrays, such as [1] and [45, 47-50], but none has considered SAA and the proposed method. EM signal measurement is conducted, and implementation of the algorithm is performed using MATLAB_R2018b software. We evaluated the performance of source localization by calculating the RMSE of range and DoA estimation experiment using the acquired data from SAA. A statistical analysis of $\mathrm{SH}$ features is equally conducted. We adopt an objective evaluation method to evaluate the outputs.

6.1. Experiment. We conducted an experiment to obtain data using an SAA $[55,63]$ in a similar way to our previous work reported in [55]. The SAA is positioned at the centre, and the source is situated at different directions and range using the centre of the SAA as reference. The elevation and azimuth of the source are fixed at $90^{\circ}$ and 0 to $360^{\circ}$, with a step size of $90^{\circ}$, respectively. The range used is between $10 \mathrm{~cm}$ and $40 \mathrm{~cm}$, with a step size of $5 \mathrm{~cm}$. EM waves emitted by an antenna were used as source signals. The performance evaluation of the proposed methods for range estimation and NF DoA estimation is performed. The comparison of performance between SH-MUSIC, SHR, SRP-PHAT, and respective proposed methods for DoA and range estimation is as summarized in Table 1. It can be seen that the proposed methods performed better than SH-MUSIC4 and SRPPHAT in the estimation of DoA and the SHR in the estimation of range.

6.2. NF DoA Estimation. The results for NF DoA estimation are given in this subsection. The performance evaluation is conducted using RMSE approach, and it is given below.

$$
\operatorname{RMSE}[0]=\sqrt{\frac{1}{G} \sum_{g=1}^{G}\left[\left(\phi_{g}-\phi \wedge_{g}\right)^{2}+\left(\theta_{g}-\theta \wedge_{g}\right)^{2}\right]},
$$

where $\left(\theta_{g}, \phi_{g}\right)$ denotes the elevation and azimuth angles of $g$ th sample and $\left(\widehat{\theta}_{g}, \widehat{\phi}_{g}\right)$ represents the corresponding estimates. $G$ is the total samples. 


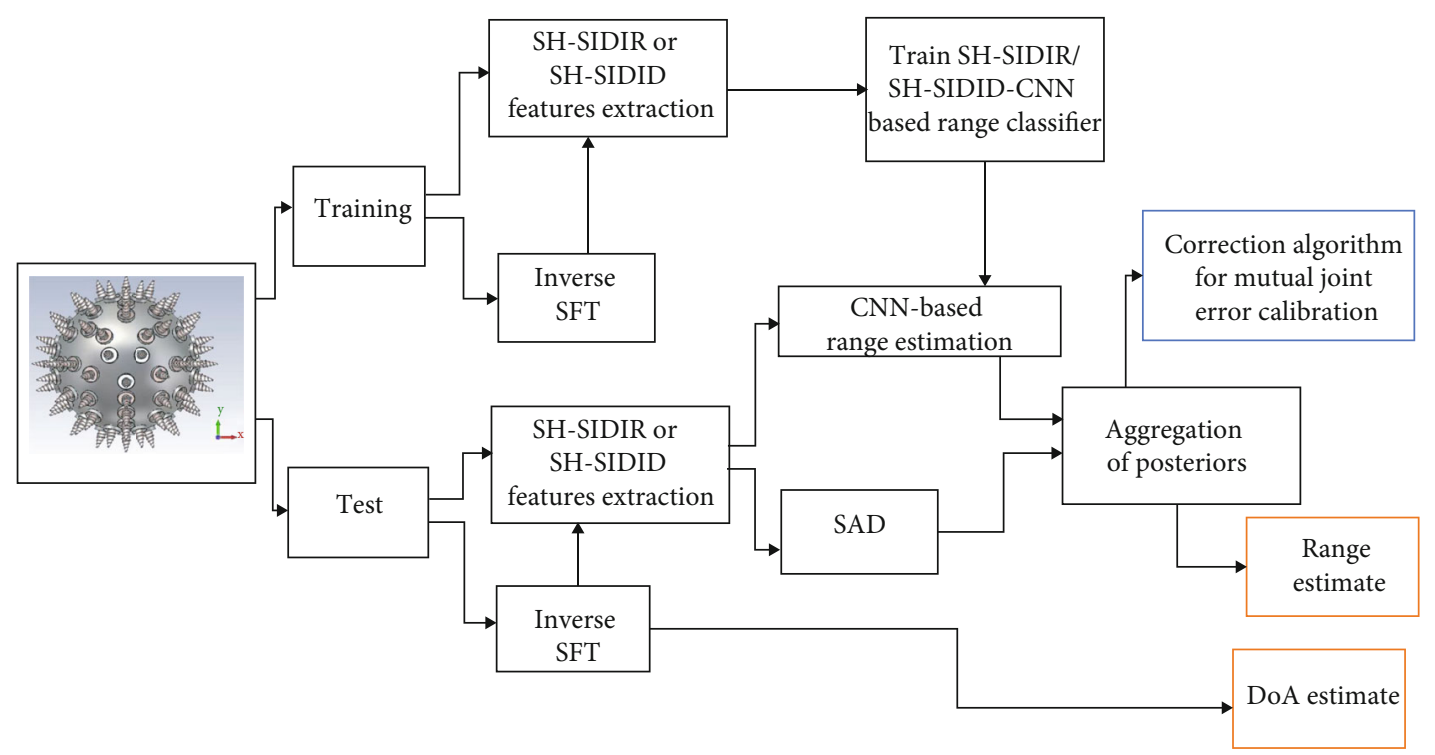

Figure 2: Procedure for NF localization of EM signals using SAA.

Requirement: $\boldsymbol{p}\left(k, r_{e}, \theta, \phi\right)$ observations, trained SAD model, and trained models for SH-SIDID/SH-SIDIR features mapped to range source.

(a) Spatial domain transformation to $\mathrm{SH}$ domain using $\boldsymbol{p}_{n m}\left(k r_{e}\right)=\boldsymbol{Y}^{\boldsymbol{H}}(\boldsymbol{\Omega}) p\left(k, r_{e}, \theta, \phi\right)$

(b) Using SFT to perform interpolation on the sphere

$$
\begin{aligned}
p\left(k, r_{e}, \theta_{e}, \phi_{e}\right)= & \sum_{n=0}^{N} \sum_{m=-n}^{n} p_{n m}\left(k r_{e}\right) Y_{n}^{m}\left(\theta_{e}, \phi_{e}\right) \\
& \forall\left(\theta_{e}, \phi_{e}\right) \in Z^{2}
\end{aligned}
$$

(c) Perform near-field DoA estimation from

$$
\left(\widehat{\theta}_{s}, \widehat{\phi}_{s}\right)=\arg \max _{\left(\theta_{e}, \phi_{e}\right)}\left|p\left(k, r_{e}, \theta_{e}, \phi_{e}\right)\right|^{2}
$$

(d) Extract SI SH features using

$$
\tilde{\boldsymbol{p}}_{n \boldsymbol{m}}\left(k r_{e}\right)=\frac{\boldsymbol{p}_{n m}\left(k r_{e}\right)}{p_{00}\left(k r_{e}\right)}
$$

(e) Extract SH-SIDID or SH-SIDIR features from

$q_{n m}\left(k r_{e}\right)=\boldsymbol{D}\left(0,-\widehat{\theta}_{s},-\widehat{\phi}_{s}\right) \tilde{\boldsymbol{p}}_{n m}\left(k r_{e}\right)$, or

$q_{n m}\left(k r_{e}\right)=\tilde{\boldsymbol{p}}_{n m}\left(k r_{e}\right) \Theta\left[\boldsymbol{Y}_{n}^{m}\left(\theta \wedge_{s}, \phi \wedge_{s}\right)\right]^{*}$ the parameters are defined in Eqns. (20) and (23)

(f) Feed the normalized $q_{n m}\left(k r_{e}\right) \forall k$ to the input of the trained CNN, which gives posterior probabilities $P_{r}\left(r_{s} \mid \boldsymbol{Q}\right)$

(g) SAD posterior probabilities implying the presence of signal are calculated.

(h) $P_{r}\left(r_{s} \mid \boldsymbol{Q}\right)$ aggregation is conducted via SAD posterior probabilities and range is consequently estimated.

(i) Obtain the complete NF source localization with the range and DoA estimates.

Algorithm 1: Proposed NF signal source localization procedure.

TABLE 1: Comparison of performance between the proposed method, SH-MUSIC, SRP-PHAT, and SHR on measured data.

\begin{tabular}{lccc}
\hline DoA estimation & & \multicolumn{2}{c}{ Range estimation } \\
Method & RMSE $\left({ }^{\circ}\right)$ & Method & RMSE $(\mathrm{cm})$ \\
\hline Inverse SFT & 15.2 & SH-SIDIR-CNN & 15.8 \\
SRP-PHAT & 51.3 & SH-SIDID-CNN & 13.4 \\
SH-MUSIC & 23.4 & SHR & 20.3 \\
\hline
\end{tabular}

We made 105 test DoAs using various azimuth and seven angles of elevation, which are selected randomly within $(0,360)^{\circ}$ and $(30,150)^{\circ}$, respectively. Signal-to-noise ratio $(\mathrm{SNR})$ is ranged between $(0,20) \mathrm{dB}$ with $5 \mathrm{~dB}$ steps. AWGN is employed in the simulations. Fourier transform with 1024 points is utilized. In each resulting dataset, estimation of DoA is conducted by 20 adjacent frames with $100 \mathrm{~ms}$ frame length and overlap of $25 \%$. The inverse SFT and SHMUSIC methods are adapted using $\mathrm{SH}$ up to $N=4$ order, while SRP-PHAT is adapted by data obtained from all the elements of SAA. The search grid is generated using 


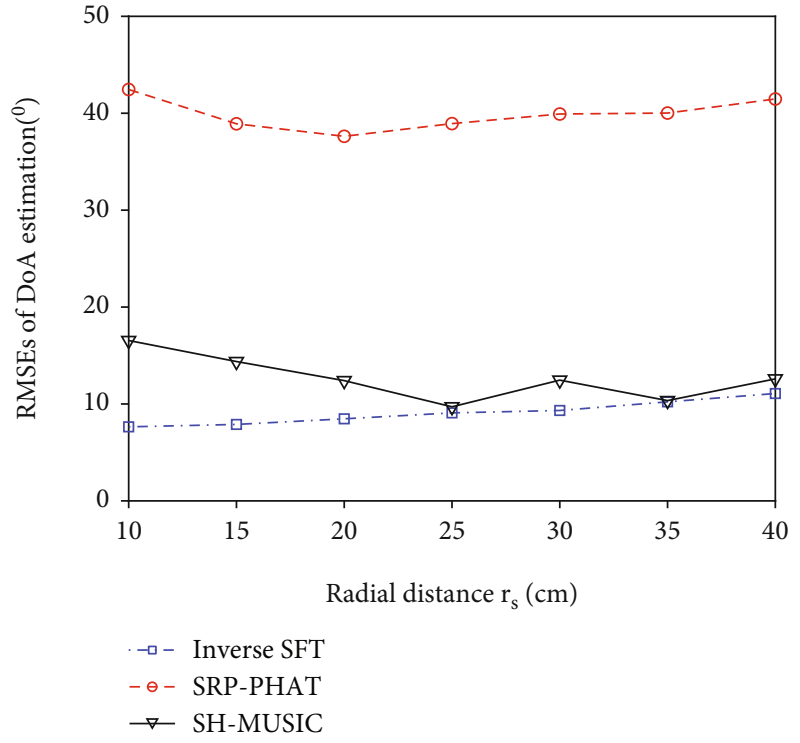

(a)

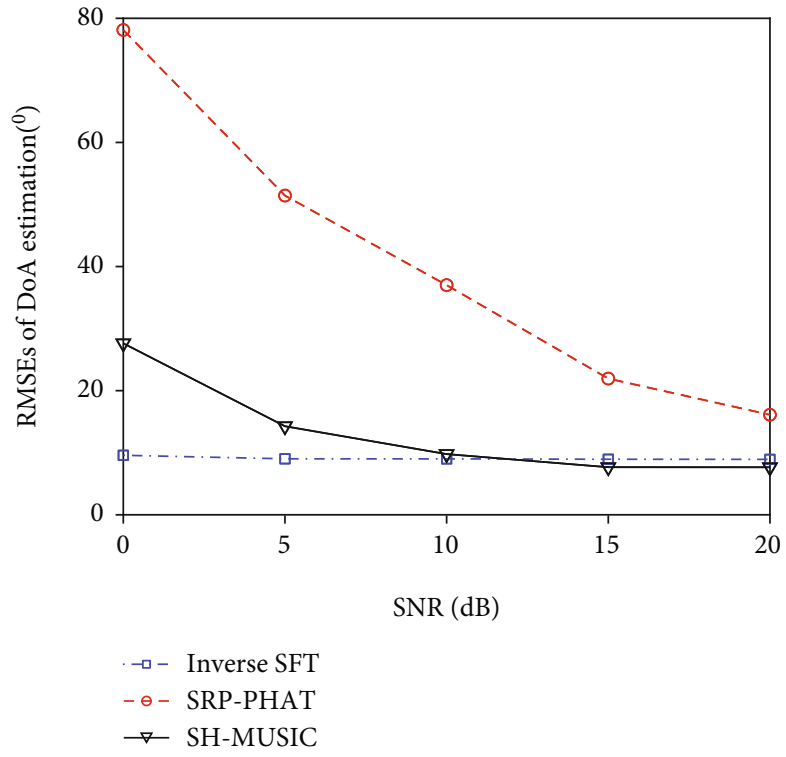

(b)

Figure 3: RMSE $\left(^{\circ}\right.$ ) of DoA estimation methods versus $r_{s}$ (a) and SNR (b). The RMSE value is the average of all possible combinations of $r_{s}$ and SNR.

TABle 2: Obtained values of $P_{\text {mean }}$ from KWT for the determination of DI and RD levels.

\begin{tabular}{lcccc}
\hline Requirement & \multirow{2}{*}{ Category } & SH-SIDIR & SH-SIDID & SHR \\
\hline Independency & DoA & 1.3082 & 1.8949 & $4 E-87$ \\
Dependency & Range & 0.7915 & 0.5497 & 0.2154 \\
\hline
\end{tabular}

uniformly sampling azimuth $(0,360)^{\circ}$ and elevation $(30$, $150)^{\circ}$, respectively. Consequently, we computed RMSE of all the methods for realizable combinations of SNR and $r_{s}$. The source distances of the SAA under consideration are proportionate to the critical distance that corresponds to EM conditions.

The comparison of performance obtained between the proposed inverse SFT for the estimation of DoA and previous methods (i.e., SRP-PHAT and SH-MUSIC) is as presented in Figure 3. It is revealed that the proposed inverse SFT method preforms better than SH-MUSIC and SRPPHAT under all conditions. Also, SH-MUSIC performs better than SRP-PHAT in all cases. When noise is increased, the performance of SH-MUSIC degrades. This is because of the spurious peaks and distortion in the magnitude spectrum of SH-MUSIC in harsh environments. With high noise, there is little or negligible performance degradation in the proposed inverse SFT. With a change in $r_{s}$, both SH-MUSIC and SRPPHAT have no particular pattern in performance. At the lower $r_{s}$, the proposed inverse SFT method performs much better, because there is a significant variation in the energy across the positions over the sphere. As $r_{s}$ increases, the energy variation between various positions on the sphere reduces; hence, inverse SFT performs better in NF. Because of the noncoincidence nature of test DoAs with search grid, the least RMSE value is calculated using the angular distance existing between test DoAs and the proximate and respective DoA available on the search grid. The lowest RMSE of the selected search grid and test DoAs configuration is $6.45^{\circ}$. This discusses the RMSEs of the proposed method in different cases. It is observed that the proposed technique achieves RMSE that is close to the minimum under $r_{s}=10 \mathrm{~cm}$, implying the better performance of the inverse SFT at lower $r_{s}$.

6.3. Statistical Discussion on SH Features. In this subsection, the discussion on SH-SIDID, SH-SIDIR, and SHR is presented using ANOVA (analysis of variance). We evaluated them under two categories: level of range dependence (RD) and level of DI. Specifically, we conducted a Shapiro-Wilk normality test [62] to know if the feature vectors are of normal distribution. Consequently, it was observed the feature vectors have no normal distribution. Therefore, a nonparametric ANOVA is performed employing a Kruskal-Wallis Test (KWT) [64, 65]. The KWT statistic $H$, as in [64], is usually approximated using chi-squared distribution [66]. Therefore, $H$ is employed to calculate $P$ value (i.e., the probability of having at least a test statistic at the extreme in a chi-squared distribution). Because the value of $P$ is calculated for various order and degrees of $\mathrm{SH}$, the mean value of $P\left(P_{\text {mean }}\right)$ is then calculated. Hence, small $P_{\text {mean }}$ value that falls under a significant level implies there is enough evidence for the rejection of the null hypothesis. Frequently, the 0.05 significant level is adapted as a benchmark between the significant and insignificant results.

Feature dependency of feature vectors is evaluated via KWT with the range as a categorical variable. The feature vectors are computed for different DoA and range combinations. The value of $P_{\text {mean }}$ presented in Table 2 shows the values of various features obtained from KWT with the 


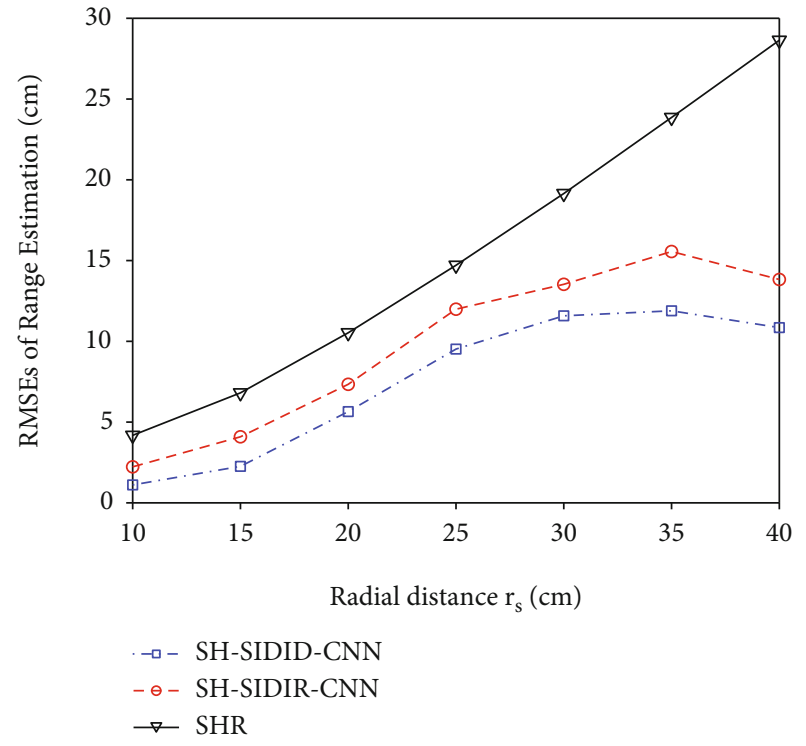

(a)

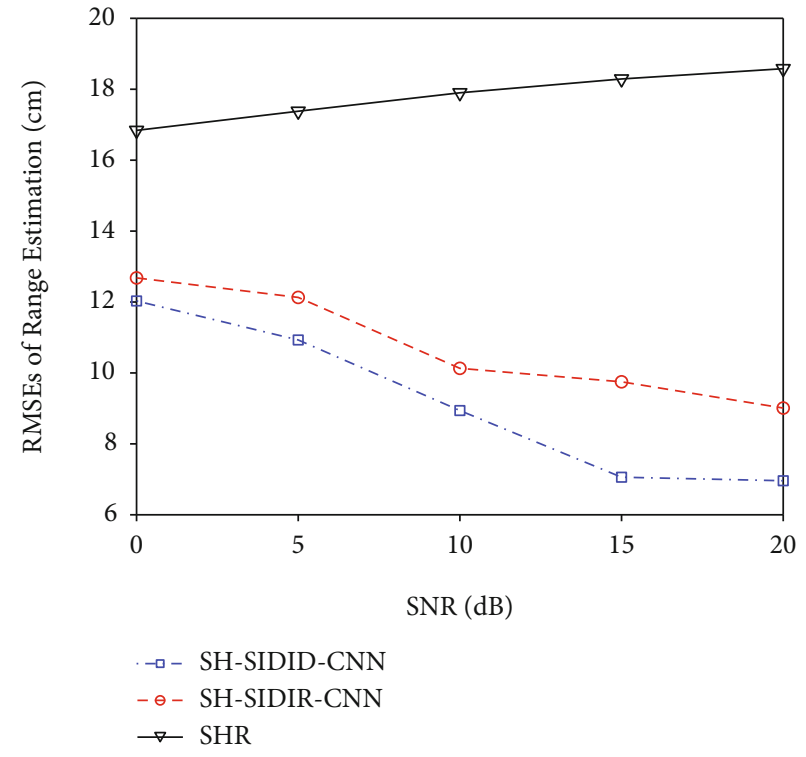

(b)

FIGURE 4: Range estimation methods versus $r_{s}$ (a) and SNR (b).

categorical variable as a range. It is observed that SH-SIDIR and SH-SIDID features have appreciable statistical information, which makes them better choices for estimation of range.

DI of various feature vectors is evaluated via KWT using DoA as a categorical variable. The computation of the feature vector was performed for different combinations of DoA while keeping the range constant. Because of the requirement of $\mathrm{DI}$, it becomes a desire to accept the null hypothesis. The values of $P_{\text {mean }}$ obtained for different features from KWT is presented in Table 2. It is seen that for SH-SIDID and SH-SIDIR features, there is acceptance of the null hypothesis, while there is rejection in the SHR feature vector. Therefore, SH-SIDI and SH-SIDIR feature vectors do not depend on the DoA in a significant manner and are better choices for the estimation of range.

6.4. Range Estimation. This subsection gives the results and discussion of range estimation. The RMSE of range estimation can be computed using

$$
\operatorname{RMSE}(\mathrm{cm})=\sqrt{\frac{1}{G} \sum_{g=1}^{G}\left[\left(r_{g}-r \wedge_{g}\right)^{2}\right]}
$$

where $r_{l}$ represents the source range of $g$ th sample and $\widehat{r}_{g}$ denotes the estimates corresponding to it.

Here, the condition of both training and testing is presented since the technique is learning dependent. For the training dataset, 200 SAA responses are taken by selecting DoA randomly and the size of the chamber for each $r_{s}$. There is a convolution of individual SAA response with 5 two-second durations of various EM signals with the random SNR to obtain five measurements. About 1024-point short time Fourier transform is employed. We randomly chose ten frames of $100 \mathrm{~ms}$ for feature extraction. Hence, the training dataset is made up of 70000 samples. For the test dataset, $50 \mathrm{SAA}$ responses are taken with random DoA, chamber size, and particular condition for each $r_{s}$. There is a convolution of each SAA response with two EM signals to get two measurements, each of which is $3 \mathrm{sec}$. The 20 corresponding $100 \mathrm{~ms}$ with $25 \%$ overlap frames are then taken from individual measurement to get the aggregate. Hence, the test dataset is made up of 14000 test samples. We have 20 samples of that test datasets for the individual combination of SNR. The resulted features are normalized and fed into the CNN. The dataset for validation is generated similar to that of the training except that the responses from SAA are 20 for each $r_{s}$. Therefore, the validation dataset is $10 \%$ of the training dataset. Note that various seeding patterns for the generation of the random number are ensured in the training, validation, and test datasets. Therefore, there is no overlap in the experimental conditions of training, validation, and test datasets.

Comparison of performance between the proposed methods and SHR in range estimation is depicted in Figure 4. The RMSE of the varying $r_{s}$ and SNR is given. It is shown how the proposed methods performed better than SHR under all scenarios. Small performance degradation is noticed in SH-SIDID-CNN and SH-SIDIR-CNN. Hence, the proposed methods show a higher level of robustness to harsh condition. It is important to note that noise has a notable effect on the performance of the proposed methods, but the performance of SHR remains almost constant. The degradation in performance of as $r_{s}$ increases could be because NF mode strengths look closer when $r_{s}$ increases. Therefore, it is not easy to differentiate between the $r_{s}$, which causes an increment in the value of RMSE as $r_{s}$ increases. 


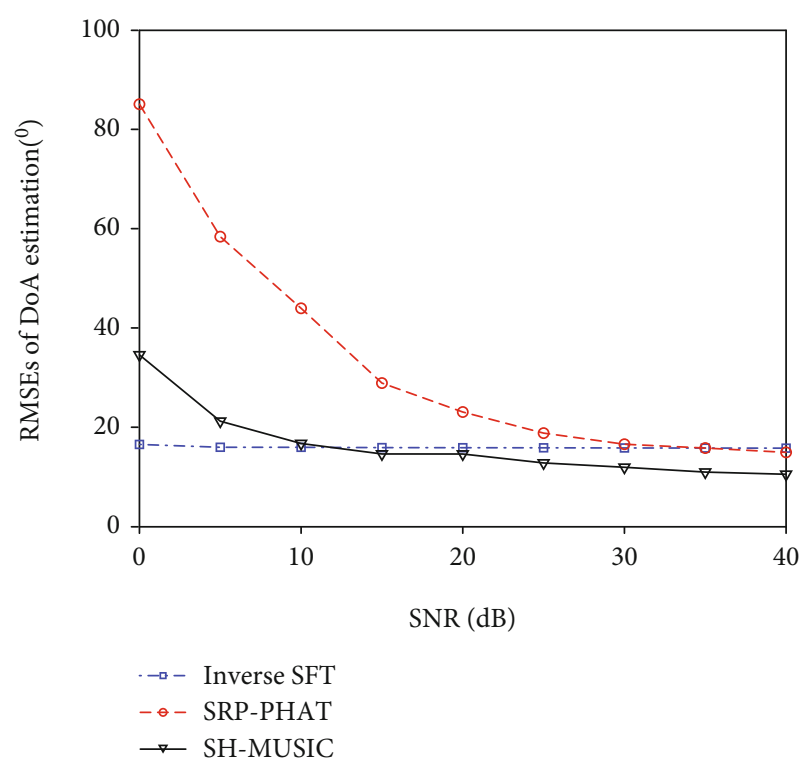

FIGURE 5: RMSE of DoA estimation methods versus SNR using measured data in [55].

Finally, fulfilling the growing application requirements, a specific number of elements are situated on the systems. The interelement distance is becoming shorter, causing strong mutual coupling with poor radiation performance and impedance matching. In order to incorporate the mutual coupling effect, which exist between elements, experimental measured data, which is the ground truth to systematically evaluate any procedure, is used. Therefore, experimental measurement data are further used for performance evaluation and analysis. The SAA is positioned in the middle of the chamber, and the source is situated at $74 \mathrm{DoAs}$, which are gotten from different combinations of 4 different elevations and 18 different azimuths. We selected the azimuths from 5 degrees to 365 degrees with 20 degrees as step size. For detailed information on measurement architecture/setup using SAA, readers are referred to the previous paper [55] where the measured data were first published. Far-field to near-field transformation is conducted on the data to conform with the current discussion. The comparison of performance between inverse SFT, SRPPHAT, and SH-MUSIC was conducted. RMSE of DoA estimation methods versus SNR is plotted, and results are as presented in Figure 5. The inverse SFT exhibits greater performance than SH-MUSIC and SRP-PHAT, even in the presence of unknown mutual coupling. Furthermore, SHMUSIC exhibits better performance than the SRP-PHAT in all scenarios. When there is increase in noise, SH-MUSIC degrades in performance. This can be attributed to the spurious peaks and distortion in the magnitude spectrum of $\mathrm{SH}-$ MUSIC when noise is high. When noise is high, there is negligible or little performance degradation in the proposed inverse SFT in the presence of mutual coupling.

\section{Conclusion}

In conclusion, a procedure for source localization of EM waves in the NF of an SAA in the presence of unknown
MC using SH features has been presented. Specifically, an approach for the estimation of NF DoA by spatial pressure interpolation through inverse SFT is proposed. Through the sampling of signal pressure at some positions on the sphere, the interpolation of signal pressure at an observation point on the sphere is facilitated using inverse SFT. The proposed procedure made use of this inverse SFT characteristic for the estimation of NF DoA. The SH features, which are SIDI, were extracted from the DoA estimate. The SI is extracted via normalization of $\mathrm{SH}$ coefficients with a part corresponding to source signal strength. The decomposition of NF steering vector into DoA-dependent and mode strength component is facilitated by $\mathrm{SH}$ decomposition. With this decomposition and DoA estimates from inverse SFT technique, we obtained the DI characteristic by two techniques. In the first technique, the $\mathrm{SH}$ rotation technique is used to extract the feature. In the second technique, there is a division of $\mathrm{SH}$ coefficients by corresponding $\mathrm{SH}$ DoA estimate. Because there is variation in the information of the range per frequency and features obtained are impaired due to harsh environment, a CNN is adapted to map the $\mathrm{SH}$ features with the range of the NF source. Finally, performance evaluation was conducted using numerical simulations and estimations from measured data, and the results obtained show that the proposed procedure exhibits appreciable performance than the previous methods, even under harsh condition. These motivating results make the proposed procedure appropriate in practical cases. The authors are currently investigating the problem of multisource localization of EM waves in the NF of SAA, and this study is planned to be presented and reported in a separate followup article.

\section{Data Availability}

The data used to support the findings of this study are available from the corresponding author upon request.

\section{Conflicts of Interest}

The authors declare that there is no conflict of interest regarding the publication of this paper.

\section{Acknowledgments}

This research is supported in part by the University Research Committee (URC) of the University of Johannesburg, South Africa.

\section{References}

[1] B. Friedlander, "Localization of signals in the near-field of an antenna array," IEEE Transactions on Signal Processing, vol. 67, no. 15, pp. 2901-2907, 2019.

[2] H. Krim and M. Viberg, "Two decades of array signal processing research: the parametric approach," IEEE Signal Processing Magazine, vol. 13, no. 4, pp. 67-94, 1996.

[3] H. L. V. Trees, Detection, Estimation, and Modulation Theory, Optimum Array Processing, Wiley, Hoboken, NJ, USA, 2004. 
[4] D. E. D. D. H. Johnson, Array Signal Processing-Concepts and Techniques, Prentice-Hall, Englewood Cliffs, NJ, USA, 1993.

[5] E. Grosicki, K. Abed-Meraim, and Y. Hua, "A weighted linear prediction method for near-field source localization," IEEE Transactions on Signal Processing, vol. 53, no. 10, pp. 36513660, 2005.

[6] Y. D. Huang and M. Barkat, "Near-field multiple source localization by passive sensor array," IEEE Transactions on Antennas and Propagation, vol. 39, no. 7, pp. 968-975, 1991.

[7] B. P. Kumar, C. Kumar, V. S. Kumar, and V. V. Srinivasan, "Reliability considerations of spherical phased array antenna for satellites," IEEE Transactions on Aerospace and Electronic Systems, vol. 54, no. 3, pp. 1381-1391, 2018.

[8] M. N. E. Korso, R. Boyer, A. Renaux, and S. Marcos, "Conditional and unconditional Cramer-Rao bounds for near-field source localization," IEEE Transactions on Signal Processing, vol. 58, no. 5, pp. 2901-2907, 2010.

[9] O. J. Famoriji, K. Akingbade, E. Ogunti, W. Apena, A. Fadamiro, and F. Lin, "Analysis of phased array antenna system via spherical harmonics decomposition," IET Communications, vol. 13, no. 18, pp. 5434-5454, 2019.

[10] G. Liu and X. Sun, "Efficient method of passive localization for mixed far-field and near-field sources," IEEE Antennas and Wireless Propagation Letters, vol. 12, pp. 902-905, 2013.

[11] I. Agrawal and R. M. Hegde, "Radial filters for near field source separation in spherical harmonic domain," in Proceedings 2016 IEEE International Conference on Acoustics, Speech and Signal Processing (ICASSP), pp. 116-120, Shanghai, China, 2016.

[12] E. Fisher and B. Rafaely, "Near-field spherical microphone array processing with radial filtering," IEEE Transactions on Audio, Speech, and Language Processing, vol. 19, no. 2, pp. 256-265, 2011.

[13] J. Meyer and G. W. Elko, "Position independent close-talking micro-phone," Signal Processing, vol. 86, no. 6, pp. 12541259, 2006.

[14] L. Kumar and R. M. Hegde, "Near-field acoustic source localization and beamforming in spherical harmonics domain," IEEE Transactions on Signal Processing, vol. 64, no. 13, pp. 3351-3361, 2016.

[15] X. Wu and J. Yan, "3-D mixed far-field and near-field source localization," IEEE Transactions on Vehicular Technology, vol. 69, no. 6, pp. 6833-6837, 2020.

[16] T. Shu, J. He, and V. Dakulagi, "3-D near-field source localization using a spatially spread acoustic vector sensor," IEEE Transactions on Aerospace and Electronic Systems, 2021.

[17] Z. Huang, B. Xue, W. Wang, F. Dong, and D. Wang, "A low complexity localization algorithm for mixed far-field and near-field sources," IEEE Communication Letters, 2021.

[18] Z. Huang, W. Wang, F. Dong, and D. Wang, "A one-snapshot localization algorithm for mixed far-field and near-field sources," IEEE Communication Letters, vol. 24, no. 5, pp. 1010-1014, 2020.

[19] Y. Kuznetsov, A. Baev, M. Konovalyuk, A. Gorbunova, and J. A. Russer, "Autocorrelation analysis and near-field localization of the radiating sources with cyclostationary properties," IEEE Transactions on Electromagnetic Compatibility, vol. 62, no. 5, pp. 2186-2195, 2020.

[20] X. Wu and J. Yan, "Gridless mixed sources localization based on low-rank matrix reconstruction," IEEE Communication Letters, vol. 9, no. 10, pp. 1748-1753, 2020.
[21] C. Guanghui, Z. Xiaoping, J. Shuang, Y. Anning, and L. Qi, "High accuracy near-field localization algorithm at low SNR using fouth-order cumulant," IEEE Communication Letters, vol. 24, no. 3, pp. 553-557, 2020.

[22] J. He, L. Li, and T. Shu, "Localization of near-field sources for exact source-sensor spatial geometry," IEEE Signal Processing Letters, vol. 27, pp. 1040-1044, 2020.

[23] H. Ma, H. Tao, and H. Kang, "Mixed far-field and near-field source localization using a linear electromagnetic-vectorsensor array with gain/phase uncertainties," IEEE Access, vol. 9, pp. 132412-132428, 2021.

[24] H. Ma, H. Tao, and J. Xie, "Mixed far-field and near-field source localization using a linear tripole array," IEEE Communication Letters, vol. 9, no. 6, pp. 889-893, 2020.

[25] J. He, L. Li, T. Shu, and T. Truong, "Mixed near-field and farfield source localization based on exact spatial propagation geometry," IEEE Transactions on Vehicular Technology, vol. 70, no. 4, pp. 3540-3552, 2021.

[26] T. Shu, L. Li, and J. He, "Near-field localization for noncircular sources in the presence of sensor phase uncertainties," IEEE Communication Letters, vol. 10, no. 3, pp. 562-566, 2021.

[27] W. Zhi and M. Y. Chia, "Near-field source localization via symmetry subarray," IEEE Signal Processing Letters, vol. 14, pp. 409-413, 2007.

[28] T. Shu, L. Li, and J. He, "Near-field source localization with two-level nested arrays," IEEE Communication Letters, vol. 24, no. 11, pp. 2488-2493, 2020.

[29] W. Zuo, J. Xin, N. Zheng, H. Ohmori, and A. Sano, "Subspacebased near-field source localization in unknown spatially nonuniform noise environment," IEEE Transactions on Signal Processing, vol. 68, pp. 4713-4726, 2020.

[30] C. Knapp and G. Carter, "The generalized correlation method for estimation of time delay," IEEE transactions on acoustics, speech, and signal processing, vol. ASSP-24, no. 4, pp. 320327, 1976.

[31] M. S. Brandstein and H. F. Silverman, "A robust method for speech signal time-delay estimation in reverberant rooms," in Proceedings 1997 IEEE International Conference on Acoustics, Speech, and Signal Processing, pp. 375-378, Munich, Germany, 1997.

[32] A. Johansson, G. Cook, and S. Nordholm, "Acoustic direction of arrival estimation, a comparison between root-music and SRP-PHAT," in Proceedings IEEE TENCON, pp. 629-632, Chiang Mai, Thailand, November 2004.

[33] L. Kumar, A. Tripathy, and R. M. Hegde, "Robust multisource localization over planar arrays using music-group delay spectrum," IEEE Transactions on Signal Processing, vol. 62, no. 17, pp. 4627-4636, 2014.

[34] V. Varanasi and R. Hegde, "Robust online direction of arrival estimation using low dimensional spherical harmonic features," in 2017 IEEE International Conference on Acoustics, Speech and Signal Processing (ICASSP), pp. 511-515, New Orleans, LA, USA, 2017.

[35] Y. Huang, J. Benesty, and G. W. Elko, “Adaptive eigenvalue decomposition algorithm for real time acoustic source localization system," in Proceedings International Conference on Acoustics, Speech, and Signal Processing, vol. 2, pp. 937-940, Phoenix, AZ, USA, 1999.

[36] C. Zhang, D. Florencio, and Z. Zhang, "Why does PHAT work well in low noise, reverberative environments?," in Proceedings 
IEEE International Conference on Acoustics, Speech and Signal Processing, pp. 2565-2568, Las Vegas, NV, USA, 2008.

[37] E. Fisher and B. Rafaely, "The nearfield spherical microphone array," in Proceedings IEEE International Conference on Acoustics, Speech and Signal Processing, pp. 5272-5275, Las Vegas, NV, USA, 2008.

[38] Q. Huang and T. Chen, "One-dimensional MUSIC-type algorithm for spherical microphone arrays," IEEE Access, vol. 8, pp. 28178-28187, 2020.

[39] W. Gao and H. Chen, "An order-aware scheme for robust direction of arrival estimation in the spherical harmonic domain," The Journal of the Acoustical Society of America, vol. 146, no. 6, p. 4883, 2019.

[40] Y. R. Zheng, R. A. Goubran, and M. El-Tanany, "Robust nearfield adaptive beamforming with distance discrimination," IEEE Transactions on Speech and Audio Processing, vol. 12, no. 5, pp. 478-488, 2004.

[41] F. Jacobsen, G. Moreno-Pescador, E. Fernandez-Grande, and J. Hald, "Near field acoustic holography with microphones on a rigid sphere (L)," The Journal of the Acoustical Society of America, vol. 129, no. 6, pp. 3461-3464, 2011.

[42] B. Rafaely, "Spatial sampling and beamforming for spherical microphone arrays," in Proceedings Hands-Free Speech Communication Microphone Arrays, pp. 5-8, Trento, Italy, 2008.

[43] S. Nakamura, "Acoustic sound database collected for handsfree speech recognition and sound scene understanding," in Proceedings International Workshop on Hands-Free Speech Communication, pp. 43-46, Baixas, France, 2001.

[44] R. Takeda and K. Komatani, "Sound source localization based on deep neural networks with directional activate function exploiting phase information," in Proceedings IEEE International Conference on Acoustics, Speech and Signal Processing, pp. 405-409, Shanghai, China, 2016.

[45] J. He, M. O. Ahmad, and M. N. S. Swamy, "Near-field localization of partially polarized sources with a cross-dipole array," IEEE Transactions on Aerospace and Electronic Systems, vol. 49, no. 2, pp. 857-870, 2013.

[46] N. Guzey, H. Xu, and S. Jagannathan, "Localization of nearfield radio controlled unintended emitting sources in the presence of multipath fading," IEEE Transactions on Instrumentation and Measurement, vol. 63, no. 11, pp. 2696-2707, 2014.

[47] Z. Zheng, M. Fu, W. Wang, S. Zhang, and Y. Liao, "Localization of mixed near-field and far-field sources using symmetric double-nested arrays," IEEE Transactions on Antennas and Propagation, vol. 67, no. 11, pp. 7059-7071, 2019.

[48] D. Sarkar, S. M. Mikki, and Y. M. M. Antar, "Poynting localized energy: method and applications to gain enhancement in coupled antenna systems," IEEE Transactions on Antennas and Propagation, vol. 68, no. 5, pp. 39783989, 2020.

[49] G. Liu and X. Sun, "Efficient method of passive localization for mixed far-field and near-field sources," IEEE Antennas and Wireless Propagation Letters, vol. 12, pp. 902-905, 2013.

[50] Z. Zheng, M. Fu, W.-Q. Wang, and H. C. So, "Symmetric displaced coprime array configurations for mixed near and far field source localization," IEEE Transactions on Antennas and Propagation, vol. 69, no. 1, pp. 465-477, 2021.

[51] E. Fisher and B. Rafaely, "Dolph-Chebyshev radial filter for the near-field spherical microphone array," in 2009 IEEE Work- shop on Applications of Signal Processing to Audio and Acoustics, pp. 169-172, New Paltz, NY, USA, 2009.

[52] H. Teutsch and G. W. Elko, "An adaptive close-talking microphone array," in Proceedings of the 2001 IEEE Workshop on the Applications of Signal Processing to Audio and Acoustics, pp. 163-166, New Paltz, New York, 2001.

[53] O. Koutny, J.-H. T. Jiricek, and M. Brothanek, "Source distance determination based on the spherical harmonics," Mechanical Systems and Signal Processing, vol. 85, pp. 9931004, 2017.

[54] V. Varanasi, A. Agarwal, and R. M. Hegde, "Near-field acoustic source localization using spherical harmonic features," IEEE/ACM Transactions on Audio, Speech, and Language Processing, vol. 27, no. 12, pp. 2054-2066, 2019.

[55] O. J. Famoriji, O. Y. Ogundepo, and X. Qi, “An intelligent deep learning-based direction-of-arrival estimation scheme using spherical antenna array with unknown mutual coupling," IEEE Access, vol. 8, pp. 179259-179272, 2020.

[56] G. Arfken and H. Weber, Mathematical Methods for Physicists, Elsevier, New York, NY, USA, 6th edition, 2005.

[57] M. Agmon and B. Rafaely, "Beamforming for a spherical-aperture microphone," in 2008 IEEE 25th Convention of Electrical and Electronics Engineers in Israel, pp. 227-230, Eilat, Israel, 2008.

[58] B. Rafaely, "Analysis and design of spherical microphone arrays," IEEE Transactions on Speech and Audio Processing, vol. 13, no. 1, pp. 135-143, 2005.

[59] S. Chakrabarty and E. A. P. Habets, "Broadband DOA estimation using convolutional neural networks trained with noise signals," in Proceedings IEEE Workshop on Applications of Signal Processing to Audio and Acoustics (WASPAA)., pp. 136140, New Paltz, NY, USA, 2017.

[60] A. Krizhevsky, I. Sutskever, and G. E. Hinton, "ImageNet classification with deep convolutional neural networks," Advances in Neural Information Processing Systems., pp. 1097-1105, 2012.

[61] C. M. Bishop, Neural Networks for Pattern Recognition, Oxford Univ. Press, London, U.K., 1995.

[62] J. Kim and M. Hahn, "Voice activity detection using an adaptive context attention model," IEEE Signal Processing Letters, vol. 25, no. 8, pp. 1181-1185, 2018.

[63] P. Kumar, C. Kumar, S. Kumar, and V. Srinivasan, “Active spherical phased array design for satellite payload data transmission," IEEE Trans. Antennas Propagat., vol. 63, no. 11, pp. 4783-4791, 2015.

[64] S. S. Shapiro and M. B. Wilk, "An analysis of variance test for normality (complete samples)," Biometrika, vol. 52, no. 3/4, pp. 591-611, 1965.

[65] W. H. Kruskal and W. A. Wallis, "Use of ranks in one-criterion variance analysis," Journal of the American Statistical Association, vol. 47, no. 260, pp. 583-621, 1952.

[66] B. Lantz, "The impact of sample non-normality on ANOVA and alternative methods," British Journal of Mathematical and Statistical Psychology, vol. 66, no. 2, pp. 224-244, 2013.

[67] H. Aoyama, "Mutual coupling matrix estimation and null forming methods for MBF antennas," IEICE Transactions on Communications, vol. E88-B, no. 6, pp. 2305-2312, 2005.

[68] Y. Peled and B. Rafaely, "Method for dereverberation and noise reduction using spherical microphone arrays," in IEEE 
International Conference on Acoustics, Speech and Signal Processing, pp. 113-116, Dallas, TX, USA, 2010.

[69] Y. LeCun, L. Bottou, Y. Bengio, and P. Haffner, "Gradientbased learning applied to document recognition," Proceedings of the IEEE, vol. 86, no. 11, pp. 2278-2324, 1998.

[70] R. V. Hogg and J. Ledolter, Engineering Statistics, Macmillan, New York, NY, USA, 1987. 